\title{
Laserterapia de baixa intensidade: Características dos clientes atendidos no serviço
}

\section{de Podiatria}

\author{
Low-level laser therapy: Characteristics of clients treated at the Clinical Podiatrics servisse \\ Terapia láser de bajo nivel: Características de los clientes atendidos en el servicio de Podiatría \\ Clínica
}

Recebido: 20/03/2021 | Revisado: 01/04/2021 | Aceito: 03/04/2021 | Publicado: 14/04/2021

\author{
Eugenio Fuentes Pérez Júnior \\ ORCID: https://orcid.org/0000-0003-4611-0443 \\ Universidade do Estado do Rio de Janeiro, Brasil \\ E-mail: eugenioperezjunior@gmail.com \\ Ariane da Silva Pires \\ ORCID: https://orcid.org/0000-0003-1123-493X \\ Universidade do Estado do Rio de Janeiro, Brasil \\ E-mail: arianepiresuerj@gmail.com \\ Madalena Joana Monteiro \\ ORCID: https://orcid.org/0000-0001-7102-5203 \\ Universidade do Estado do Rio de Janeiro, Brasil \\ E-mail: madajoanam@gmail.com \\ Katiana de Moraes \\ ORCID: https://orcid.org/0000-0002-6692-819X \\ Universidade do Estado do Rio de Janeiro, Brasil \\ E-mail: Moraes.Katiana@gmail.com \\ Luciano Domingos Santos \\ ORCID: https://orcid.org/0000-0002-2374-8770 \\ Universidade do Estado do Rio de Janeiro, Brasil \\ E-mail: luciano.domingos.santos@hotmail.com \\ Juilia Mônica Marcelino Benevides \\ ORCID: https://orcid.org/0000-0002-4410-1431 \\ Universidade do Estado do Amazonas, Brasil \\ E-mail: benevides.julia@gmail.com \\ Vânia Lima Coutinho \\ ORCID: https://orcid.org/0000-0001-9300-3697 \\ Universidade do Estado do Rio de Janeiro, Brasil \\ E-mail: vlcoutto@hotmail.com \\ Francisco Gleidson de Azevedo Gonçalves \\ ORCID: https://orcid.org/0000-0002-6468-8137 \\ Universidade do Estado do Rio de Janeiro, Brasil \\ E-mail: gleydy_fran@hotmail.com
}

\begin{abstract}
Resumo
Objetivo: Descrever e analisar as características dos clientes submetidos a laserterapia de baixa intensidade acompanhados em um serviço de Podiatria Clínica. Método: Estudo descritivo, retrospectivo e documental, realizado em um serviço de Podiatria Clínica no Estado do Rio de Janeiro, no período de janeiro de 2019 a janeiro de 2020. Foram analisados 110 prontuários de clientes atendidos no serviço. Resultados: Identificou-se que foram realizadas no período de 12 meses 322 sessões de laserterapia de baixa intensidade com em média 3 aplicações por cliente. Houve prevalência do sexo feminino (60\%), e faixa etária de 50 e 69 anos (54\%). Os principais diagnósticos podiátricos foram de onicomicoses (75\%); tínea pedis (15\%) e feridas (5\%). Em relação a comorbidades, $29 \%$ eram portadores de hipertensão arterial; $17 \%$ dislipidemia; e 6\% obesidade. Conclusão: O estudo possibilitou o conhecimento acerca das características clínicas da amostra estudada. Recomenda-se que mais estudos sejam realizados para disseminação do conhecimento nas áreas de Laserterapia e Podiatria Clínica que ainda são embrionárias no Brasil.
\end{abstract}

Palavras-chave: Lasers; Podiatria; Diabetes Mellitus; Enfermagem; Prática avançada de enfermagem.

\begin{abstract}
Objective: To describe and analyze the characteristics of clients undergoing low-level laser therapy followed up in a Clinical Podiatrics service. Method: Descriptive, retrospective and documentary study, carried out in a Clinical Podiatrics service in the State of Rio de Janeiro, from January 2019 to January 2020. 110 medical records of clients treated at the service were analyzed. Results: It was identified that 322 low-intensity laser therapy sessions were carried out over a 12-month period, with an average of 3 applications per client. There was a prevalence of females
\end{abstract}


(60\%), and the age group of 50 and 69 years (54\%). The main podiatric diagnoses were onychomycosis (75\%); pedis (15\%) and wounds (5\%). Regarding comorbidities, $29 \%$ had arterial hypertension; 17\% dyslipidemia; and 6\% obesity. Conclusion: The study provided knowledge about the clinical characteristics of the studied sample. It is recommended that further studies be carried out to disseminate knowledge in the areas of Laser Therapy and Clinical Podiatrics that are still embryonic in Brazil.

Keywords: Lasers; Podiatry; Diabetes Mellitus; Nursing; Advanced practice nursing.

\section{Resumen}

Objetivo: Describir y analizar las características de los clientes en tratamiento con láser de baja intensidad seguidos en un servicio de Podiatría Clínica. Método: Estudio descriptivo, retrospectivo y documental, realizado en un servicio de Podiatría Clínica en el Estado de Río de Janeiro, de enero de 2019 a enero de 2020. Se analizaron 110 historias clínicas de clientes atendidos en el servicio. Resultados: Se identificó que se realizaron 322 sesiones de terapia con láser de baja intensidad en un período de 12 meses, con un promedio de 3 aplicaciones por cliente. Hubo una prevalencia de mujeres (60\%) y el grupo de edad de 50 y 69 años (54\%). Los principales diagnósticos podiátricos fueron onicomicosis $(75 \%)$; pedis $(15 \%)$ y heridas (5\%). En cuanto a las comorbilidades, el $29 \%$ tenía hipertensión arterial; $17 \%$ de dislipidemia; y $6 \%$ de obesidad. Conclusión: El estudio proporcionó conocimiento sobre las características clínicas de la muestra estudiada. Se recomienda que se realicen más estudios para difundir el conocimiento en las áreas de Terapia Láser y Podiatría Clínica que aún se encuentran en estado embrionario en Brasil.

Palabras clave: Rayos láser; Podiatría; Diabetes Mellitus; Enfermería; Enfermería de práctica avanzada.

\section{Introduçãa}

Este estudo tem por objeto as características dos clientes submetidos a laserterapia de baixa intensidade acompanhados em um serviço de Podiatria Clínica de unidade ambulatorial especializada no Estado do Rio de Janeiro.

A laserterapia consiste na aplicação da luz por meio de uma radiação eletromagnética com alta intensidade de energia, estudada por Einstein desde 1917 através de seu experimento fotoelétrico, e incorporada como instrumento terapêutico biomédico a partir de 1960 (Almeida, Pinheiro, \& Soares, 2017).

O laser pode ser classificado de acordo com sua potência em: alta potência que emite radiação ionizante e efetua alteração estrutural no tecido, sendo utilizado para remoção de tecidos, corte e coagulação e o de baixa potência cuja a radiação é não ionizante e sua ação se dá por meio de fotobiomodelação celular contribuindo nos processo celulares e teciduais como cicatrização, no controle de infecções teciduais, na promoção de analgesia, na drenagem de edemas e no controle do processo inflamantório (Macedo, Mota, Fagundes, Souza \& Navarro 2021).

No processo de reparação recidual pode ser utilizado para acelerar e melhorar a qualidade do processo regenerativo, através de uma sequência de eventos fisiológicos e bioquímicos com a diminuição da inflamação, estimulação da síntese de colágeno, a formação do tecido de granulação e a reepitelização. Tais efeitos podem ser obtidos por meio da transferência de energia fotônica que absorvida pela célula, potencializa a produção de adenosina trifosfato (ATP) essencial as atividades metabólicas, como proliferação celular, síntese de colágeno e aceleração do processo de reparação do tecido em animais e humanos (Macedo, Mota, Fagundes, Souza \& Navarro 2021).

No que tange ao controle de infecção tecidual e proliferação celular desordenada a laserterapia pode promover controle do crescimento desordenado de células nos tecidos tumorais e fotoinativação microbiana produzida pela terapia fotodinâmica (TFD) (Liang, Lu, Chen \& Lin, 2016).

A TFD consiste na administração tópica de fotossensibilizador, corante não tóxico (azul de metileno), em conjunto com a aplicação de laser seletiva em uma lesão-alvo (fungos e bactérias), com comprimento de onda apropriada, objetivando proporcionar foto-lesão oxidativa localizada. Através da luz os fotossensibilizantes são ativados transferindo energia ao oxigênio molecular, e como resultado desta combinação, ocorre a morte do microrganismo imediata. A destruição de bactérias e fungos ocorre devido a transferência de elétrons ou hidrogênio, levando à produção de radicais livres (reação do tipo I) ou por transferência de energia ao oxigênio (reação do tipo II), induzindo a produção do oxigênio singlete, radical livre que promove a morte do patógeno (Liang, Lu, Chen \& Lin, 2016). 
Dentre as principais infecções encontradas nos pés dos idosos, portadores de doenças crônicas como o Diabetes, destacam-se as onicomicoses e tínea pedis pois são as de maior prevalência. Nessa população a incidência das onicomicoses é de $(45,9 \%)$ acometendo as áreas unilaterais dos pés e as áreas bilaterais afetadas em (20\%), enquanto a tínea pedis atinge cerca de (55\%) dos membros inferiores (Santos \& Duarte, 2019).

Acrescenta-se que a ocorrência de infecções fúngicas em pessoas com Diabetes Mellitus é um fator de risco para o desenvolvimento da úlcera de pé diabético. Portadores de DM apresentam 25\% maior chance de desenvolver ulcerações, se comparado aos não diabéticos. Destaca-se que as ulcerações causam cerca de $85 \%$ das amputações não-traumáticas dos membros inferiores, portanto, o controle de infecções fúngicas nos pés de idosos diabéticos é estratégico para a prevenção de amputações (Silva, Haddad, Rossanus, Vannuchi, \& Marcon, 2017).

Devido as suas propriedades, a laserterapia de baixa intensidade constitui-se uma importante tecnologia em saúde. No serviço de Podiatria Clínica, em particular, tem se mostrado uma importante ferramenta terapêutica empregada no cuidado de enfermagem pois possui ampla aplicabilidade tanto no tratamento quanto na reabilitação de pacientes portadores de patologias nos membros inferiores (Paula, Silva \& Faria, 2012).

Cabe ressaltar que a Podiatria despontou no Brasil a partir de 2007 com a criação do $1^{\circ}$ Curso de Especialização, na Universidade Federal de São Paulo (UNIFESP) para graduados em enfermagem. A nível mundial originou-se dos quiropodistas, primeiros profissionais relacionados ao tratamento dos pés e tornozelos. Em 1911, o Dr. Maurice J. Lewi, criou a primeira escola de Quiropodia, intitulada mais tarde como Faculdade de Nova Iorque de Medicina Podiátrica, consagrando o nome Podiatria para denominar a prática relacionada ao tratamento das patologias dos membros inferiores (Portal do Enfermeiro Podiatra Brasileiro, 2020).

Ressalta-se que no Brasil o enfermeiro habilitado ou especialista em Podiatria Clínica possui protagonismo incontestável na avaliação dos membros inferiores com o objetivo de promover a saúde por meio do diagnóstico precoce dos sinais e sintomas de processos que sejam capazes de afetar a capacidade de mobilização, assim como, complicações decorrentes de doenças agudas ou crônicas, minimizando as implicações que possam resultar em possíveis amputações (Sociedade Brasileira de Diabetes,2020).

Ao se considerar a contemporaneidade da Podiatria Clínica no Brasil e em especial a escassez de estudos e publicações na área de enfermagem no que concerne à atuação do enfermeiro tanto na área de laserterapia quanto no atendimento podiátrico, o conhecimento das características clínicas dos pacientes atendidos em um serviço de Podiatria Clínica conveniado ao Sistema Único de Saúde que utiliza tecnologias como a laserterapia de baixa intensidade enquanto terapia complementar no cuidado das patologias dos membros inferiores é relevante para subsidiar a organização e o desenvolvimento de estudos na área.

Neste contexto, o presente estudo tem como objetivo descrever e analisar as características dos clientes submetidos a laserterapia de baixa intensidade em um serviço de Podiatria Clínica.

\section{Metodologia}

Estudo descritivo, retrospectivo e documental, de abordagem quantitativa (Estrela, 2018), sobre as características dos clientes submetidos a laserterapia de baixa intensidade em um serviço de Podiatria Clínica no Estado do Rio de Janeiro, atendidos no período de janeiro de 2019 a janeiro de 2020.

O cenário de estudo foi um serviço de enfermagem especializado em Podiatria Clínica de um centro ambulatorial de referência para atendimento de clientes diabéticos no Estado do Rio de Janeiro.

Adotou-se os seguintes critérios inclusão: prontuários de clientes atendidos no serviço de Podiatria Clínica disponíveis e que continham informações acerca das características clínicas dos pacientes submetidos a laserterapia de baixa intensidade. 
Foram excluídos prontuários de pacientes que estavam incompletos e não continham informações clínicas dos pacientes ou que estavam indisponíveis no momento da coleta de dados.

A coleta de dados ocorreu nos meses de março a maio de 2020 por meio da análise de prontuários e impressos próprios do setor, referentes às características dos pacientes submetidos a laserterapia de baixa intensidade no serviço de Podiatria Clínica.

A população inicial do estudo foi composta por 123 prontuários, após aplicação dos critérios de exclusão, foram descartados do estudo 13 prontuários por não possuírem informações registradas e a amostra final constituiu-se de 110 prontuários. Admitiu-se para essa amostra índice de confiança de 95\% e erro amostral em torno de 5\%.

O impresso utilizado para esse procedimento foi preenchido, pelos enfermeiros que realizam a laserterapia de baixa intensidade. Para este estudo as variáveis avaliadas foram: sexo, idade, diagnóstico médico, conforme Classificação Internacional de Doenças-CID 10, indicação do uso da laserterapia, número de sessões de laserterapia realizadas.

Os dados após coletados foram digitados e tabulados eletronicamente e, sequencialmente, realizou-se a consolidação no programa Microsoft Excel, com análise estatística descritiva simples, e de distribuição de frequências dos dados que representaram as variáveis do estudo. Os resultados foram discutidos à luz da literatura científica disponível.

Para atender aos critérios éticos, a pesquisa foi submetida ao Comitê de Ética em Pesquisa da instituição e aprovado sob o parecer CAAE $\mathrm{n}^{\mathrm{o}}$ 16427419.3.0000.5259. Por se tratar de uma pesquisa retrospectiva de análise documental do prontuário e impressos do próprios do setor, dispensa a assinatura do Termo de Consentimento Livre e Esclarecido.

\section{Resultados}

Após a análise dos dados identificou-se que foram realizadas no período de 12 meses 322 sessões de laserterapia de baixa intensidade com em média 3 aplicações por clientes atendidos nesse mesmo período.

No que tange ao sexo dos clientes tratados verificou-se que a amostra apresenta predomínio do sexo feminino como descrito na Tabela 1.

Tabela 1 - Pacientes atendidos no serviço por sexo, Rio de Janeiro, 2020.

\begin{tabular}{lll}
\hline Sexo & $\mathbf{n}^{\mathbf{0}}$ & $\%$ \\
\hline Masculino & 44 & $40 \%$ \\
\hline Feminino & 66 & $60 \%$ \\
\hline Total & 110 & $100 \%$ \\
\hline
\end{tabular}

Fonte: Autores (2020).

Em relação a faixa etária dos clientes houve prevalência de adultos a partir de 50 anos e idosos, de acordo com a Tabela 2. 
Research, Society and Development, v. 10, n. 4, e36610414099, 2021

(CC BY 4.0) | ISSN 2525-3409 | DOI: http://dx.doi.org/10.33448/rsd-v10i4.14099

Tabela 2 - Idade por faixa etária dos clientes atendidos. Rio de janeiro, 2020.

\begin{tabular}{lcc}
\hline Faixa etária & $\mathrm{n}^{\mathbf{o}}$ & $\%$ \\
\hline 30 a 49 anos & 3 & $3 \%$ \\
\hline 50 a 69 anos & 60 & $54 \%$ \\
\hline 70 a 89 anos & 45 & $41 \%$ \\
\hline Mais de 90 anos & 2 & $2 \%$ \\
\hline Total & 110 & $100 \%$ \\
\hline
\end{tabular}

Fonte: Autores (2020).

Ao se investigar nesse grupo a indicação do uso da laserterapia de baixa intensidade detectou-se predominância de utilização para controle e tratamento auxiliar das infecções fúngicas, destacando-se as onicomicoses e tínea pedis, conforme Tabela 3.

Tabela 3 - Indicação do uso do laser, Rio de Janeiro, 2020.

\begin{tabular}{lcc}
\hline Indicação & $\mathrm{n}^{\mathbf{0}}$ & $\%$ \\
\hline Onicomicose & 242 & $75 \%$ \\
\hline Tínea Pedis & 54 & $17 \%$ \\
\hline Feridas & 17 & $5 \%$ \\
\hline Analgesia & 5 & $2 \%$ \\
\hline Drenagem linfática & 4 & $1 \%$ \\
\hline Total & 322 & $100 \%$ \\
\hline
\end{tabular}

Fonte: Autores (2020).

Considerando-se a presença de patologias, além do diabetes, o histórico de comorbidades do grupo analisado demonstrou predomínio da hipertensão arterial sistêmica, seguido de dislipidemia e obesidade como demonstrado na Tabela 4. 
Research, Society and Development, v. 10, n. 4, e36610414099, 2021

(CC BY 4.0) | ISSN 2525-3409 | DOI: http://dx.doi.org/10.33448/rsd-v10i4.14099

Tabela 4 - Diagnósticos médico dos clientes atendidos neste serviço de Podiatria Clínica.

\begin{tabular}{|c|c|c|}
\hline Diagnóstico médico & $\mathrm{n}^{\mathrm{o}}$ & $\%$ \\
\hline Hipertensão & 96 & $29,7 \%$ \\
\hline Dislipidemia & 57 & $17,6 \%$ \\
\hline Obesidade & 22 & $6,8 \%$ \\
\hline Hipotiroidismo & 20 & $6,2 \%$ \\
\hline Insuficiência renal crônica & 18 & $5,6, \%$ \\
\hline Doença arterial coronariana & 17 & $5,3 \%$ \\
\hline Doenças osteomusculares & 16 & $5,0 \%$ \\
\hline Neuropatia periférica & 15 & $4,6 \%$ \\
\hline Doença arterial periférica & 11 & $3,4 \%$ \\
\hline Doenças hematólogicas & 8 & $2,5 \%$ \\
\hline Doenças SNC & 7 & $2,2 \%$ \\
\hline Doenças hepaticas & 6 & $1,9 \%$ \\
\hline Insuficiência vascular & 6 & $1,9 \%$ \\
\hline Neoplasias & 6 & $1,9 \%$ \\
\hline Glaucoma & 5 & $1,5 \%$ \\
\hline Asma & 4 & $1,2 \%$ \\
\hline Fibrilação atrial & 4 & $1,2 \%$ \\
\hline Insuficiência cardíaca & 3 & $0,9 \%$ \\
\hline AVE isquêmico & 2 & $0,2 \%$ \\
\hline Total & 323 & $100 \%$ \\
\hline
\end{tabular}

Fonte: Autores (2020).

\section{Discussão}

As características dos clientes submetidos a laserterapia de baixa intensidade, no serviço investigado, revelam um predomínio do sexo feminino, no entanto, cabe ressaltar que no setor de Podiatria Clínica são atendidos exclusivamente pacientes com diabetes e suas complicações. Nesse contexto, encontra-se perfil similar aos serviços de ambulatórios especializados, nos quais $60 \%$ dos pacientes com Diabetes Mellitus são do sexo feminino como no registro do sistema 
epidemiológico do Sistema Único de Saúde, SIS-Hiperdia. Tal condições está relacionada a maior probabilidade de desenvolver o DM gestacional e maior prevalência de resistência insulínica relacionada ao envelhecimento e ao climatério (Palmeira \& Pinto, 2015).

Outro aspecto relevante a ser considerado ao se analisar a maior frequência de mulheres submetidas a laserterapia no serviço de podiatria, foi o fato de que as mulheres, em virtude de uma maior preocupação com a saúde, buscam assistência mais precocemente e com maior frequência que os homens com 1,4 vezes mais chances de utilizar os serviços de saúde do que o sexo oposto (Meirelles, 2015).

No que concerne à faixa etária dos indivíduos, identificou-se a predominância de indivíduos na faixa de 50 a 69 anos de idade. Seguindo a prevalência do DM, o perfil de idade identificado foi semelhante aos estudos relacionados a patologia que detectaram que a maioria de portadores de DM encontrava-se entre as idades superiores aos 40 anos (Sarno, Bittencourt \& Oliveira, 2020).

Ressalta-se que o envelhecimento da população, a crescente manutenção da obesidade e do sedentarismo e os processos de urbanização são considerados os principais fatores relacionados ao aumento da incidência e prevalência do DM tipo 2, que atinge aproximadamente $90 \%$ de todos os casos de diabetes no mundo, ao passo que o DM tipo 1, corresponde a 10\% dos casos da população mundial (Flor \& Campos , 2017).

É relevante considerar que o processo do envelhecimento é dinâmico, progressivo e fisiológico, acompanhado de modificações morfológicas e funcionais, que podem ser exacerbadas na presença do DM, como as ocorridas nas estruturas anatômicas e fisiológicas dos pés em decorrência da neuropatia periférica e/ou doença arterial periférica (DAP) que tornam os idosos diabéticos mais vulneráveis a complicações, tais como: transtornos da marcha, traumas, perda da integridade das unhas, da pele, dos nervos, dos vasos e das estruturas ósseas (Flor \& Campos , 2017).

Dentre estas complicações, destaca-se o pé diabético, caracterizado como infecção, ulceração e/ou destruição de tecidos moles associadas a alterações neurológicas e vários graus de doença arterial periférica. Essa complicação do DM está relacionada em $90 \%$ dos casos com a presença de neuropatia. A neuropatia periférica é a alteração da sensibilidade dos pés que leva a uma disfunção dos nervos periféricos, tornando o indivíduo suscetível ao desenvolvimento de traumas e ulcerações em membros inferiores (Sociedade Brasileira de Diabetes, 2020). Essa complicação é resultado de processos patológicos crônicos e portanto é, mais comum de ser encontrado em pacientes na faixa etária superior aos 40 anos.

No que diz respeito às principais indicações para o tratamento por meio da laserterapia de baixa intensidade destacamse a onicomicose e a tínea pedis, como as infecções fúngicas mais frequentemente encontradas nos pés dos idosos. A onicomicose é uma dermatofitose causada por fungos que possuem tropismo pela queratina, proteína presente na composição da lâmina ungueal e está relacionada com ambientes úmidos, quentes e escuros (ambiente facilmente encontrado no interior dos sapatos), sendo assim, os pés são as áreas mais afetadas e o hálux o principal pododáctilo atingido (Akkus et al.,2016)

O DM promove alterações que contribuem para diminuição da circulação e da resposta imunológica do indivíduo, por esse motivo, a prevalência de onicomicose e a incidência de infecções secundárias são até três vezes maiores do que na população não diabética (Sociedade Brasileira de Diabetes, 2020).

As onicomicoses possuem prevalência mundial entre $2 \%$ a $50 \%$ e representam $30 \%$ das infecções micóticas superficiais. No Brasil, segundo o Observatório Nacional de Onicomicose, ao analisar 7.852 pacientes de diversos estados encontrou a prevalência de $28 \%$ (Kallis \& Tosti, 2016).

O tratamento e controle da onicomicose por meio da laserterapia com TFD é uma das opções que apresenta eficácia comprovada mundialmente e se destaca por um custo-benefício viável (Ma et al., 2016).

Em metanálise sobre os efeitos curativos e a segurança do tratamento a laser para onicomicose, no qual foram incluídos 35 estudos, verificou-se a eficácia deste tratamento em todos os casos, com o benefício de menor efeito colateral 
quando comparado com as demais terapias farmacológicas sistêmica (Ma et al., 2016).

Acrescenta-se que laserterapia de baixa intensidade constitui-se em uma tecnologia complementar que o enfermeiro com formação específica pode utilizar como uma possibilidade terapêutica eficaz, no tratamento de infecções fúngicas por meio da TFD, com segurança e conforto. Além de apresentar melhora do aspecto da lâmina ungueal, o que corrobora com sua efetividade (Gonçalves et al., 2020).

Outra frequente utilização da laserterapia de baixa intensidade identificada foi sua aplicabilidade como recurso terapêutico complementar na cicatrização de feridas complexas. As ulcerações nos membros inferiores em pacientes com DM ocorrem em virtude das alterações vasculares, neurológicas e traumáticas, e são responsáveis pela maioria das amputações dos membros inferiores (Senteio, Teston, Costa, Soares \& Spigolon, 2018).

A laserterapia de baixa intensidade tem se mostrado um recurso terapêutico eficaz na promoção da recuperação de tecidos, favorecendo o processo de cicatrização, estímululando a formação da microcirculação, por seus efeitos antinflamatórios, antiedematosos e analgésicos (Macedo, Mota, Fagundes, Souza \& Navarro 2021).

Outras indicações para o uso da laserterapia de baixa intensidade apresentada em nosso estudo, porém de forma reduzida, foram a analgesia (2\%) e drenagem linfática (1\%). Em revisão sistemática realizada para analisar os efeitos da laserterapia de baixa intensidade na cicatrização de úlceras nos pés em pessoas com DM evidenciou que além de reduzir o tempo de tratamento, o laser pode favorecer alívio no estado álgico e no desconforto local. Outro fator importante é que um dos efeitos atribuídos à fototerapia com laser de baixa intensidade é o aumento de drenagem linfática que acarretará na aceleração da cicatrização de feridas e redução de edemas (Schmidt \& Pereira, 2017).

No que se refere aos diagnósticos na amostra estudada a hipertensão se destaca como a mais frequente, corroborando com a Sociedade Brasileira de Cardiologia que afirma que a hipertensão arterial sistêmica e o diabetes mellitus são as doenças mais frequentes em idosos, e a incidência acompanha o aumento da idade em ambos os sexos, atingindo taxas mais altas nas mulheres idosas (Picon et al., 2017).

Estudo nacional revelou que a prevalência de hipertensão e diabetes na população acima de 18 anos foi de 25,7\% e $8,9 \%$ respectivamente. Em cerca de 50\% dos pacientes a hipertensão arterial está presente e atrelada a outras comorbidades (Flor \& Campos,2017).

Outro aspecto importante relacionado ao DM tipo 2 é que os pacientes apresentam comomitantemente dislipidemia, ocasionando posteriormente doenças cardiovasculares. Acrescenta-se o fato de que os pacientes diabéticos com aterosclerose estão sujeitos de duas a quatro vezes mais aos riscos de doenças cardiovasculares quando comparados a pacientes não diabéticos. Os indivíduos que apresentam hipertensão arterial e diabetes mellitus apresentaram maior prevalência de dislipidemias (Kautzky-Willer, Harreiter \& Pacini, 2016).

A maioria das pessoas que tem diabetes mellitus tipo 2 apresenta algum grau de obesidade ou sobrepeso, tais condições contribuem para o estabelecimento da resistência insulínica. A obesidade precisa ser tratada para que a síndrome metabólica, o diabetes e a doença cardiovascular não progridam. A obesidade é um fator de risco para o diabetes já que favorece um estado de inflamação crônica e de resistência à insulina, principalmente em idosos (Sociedade Brasileira de Diabetes, 2020).

Segundo a Sociedade Brasileira de Diabetes, caso não haja controle adequado da taxa de glicemia, há o risco de surgimento de algumas complicações, principalmente nos rins, olhos, nervos, coração, vasos sanguíneos, pele e membros inferiores. Estudos epidemiológicos atestam uma ligação direta e independente entre os níveis sanguíneos de glicose e a doença cardiovascular. Do mesmo modo, há associação entre o aumento da mortalidade e ao alto risco de desenvolvimento de complicações micro e macro vasculares, bem como de neuropatias (Sociedade Brasileira de Diabetes, 2020).

A neuropatia periférica provoca a perda da sensibilidade protetora e problemas motores, estes problemas ampliam a 
chance do aparecimento de feridas nos pés devido à diminuição das sensações de dor e temperatura. Estudo descreve que aproximadamente $50 \%$ dos pacientes com diabetes mellitus desenvolvem neuropatia periférica sintomática dentro de 25 anos após o início da doença (Pimentel \& Marques,2019).

Outro diagnóstico presente em nosso estudo foi o da doença arterial periférica (3,4\%), o DM tipo 2 é considerado o principal fator de risco para a ocorrência desta condição clínica. Um estudo transversal, multicêntrico nacional com 1.170 indivíduos apresentou uma taxa de prevalência de doença arterial periférica de 10,5\% (Nunes et al., 2002).

\section{Conclusão}

A análise das características dos clientes atendidos em um serviço de Podiatria Clínica submetidos a laserterapia de baixa intensidade revelou a predominância do sexo feminino. A faixa etária mais prevalente situou-se acima dos 50 e 79 anos. Em relação às afecções dos membros inferiores, evidenciou-se maior ocorrência das onicomicoses, tínea pedis e feridas. Dentre as comorbidades mais prevalentes associadas a diabetes destacam se a hipertensão arterial sistêmica, dislipidemia e obesidade.

Ressalta-se que, a produção sobre a assistência de enfermagem nas áreas de Podiatria Clínica e Laserterapia de baixa intensidade, é escassa, devido ao baixo quantitativo de publicações. O número de enfermeiros habilitados/especializados em Podiatria Clínica no Brasil, é pequeno, bem como, os capacitados na prática da laserterapia de baixa intensidade. É possível recomendar que mais estudos sejam realizados, a fim de que o conhecimento seja disseminado nessas áreas de saber, contribuindo assim para uma assistência de enfermagem qualificada e que possa de fato contribuir para o tratamento das afecções dos pés de pacientes diabéticos e reduzir as complicações e quantitativo de amputações nessa população. Sugere-se a realização de estudos clínicos sobre a utilização da laserterapia em pacientes diabéticos.

\section{Referências}

Akkus, G., Evran, M., Gungor, D., Karakas, M., Sert, M., \& Tetiker, T. (2016). Tinea pedis and onychomycosis frequency in diabetes mellitus patients and diabetic foot ulcers. A cross sectional - observational study. Pakistan journal of medical sciences, 32(4), 891-895. https://doi.org/10.12669/pjms.324.10027

Almeida F. P, Pinheiro B. L. A \& Soares, P. G. L. Princípios fundamentais dos lasers e suas aplicações. Biotecnologia Aplicada a Agro \& Indústria. Blucher. 815-894.

Estrela, C. (2018). Metodologia Científica: Ciência, Ensino, Pesquisa. Editora Artes Médicas.

Flor, L. S. \& Campos, M. R. (2017). Prevalência de diabetes mellitus e fatores associados na população adulta brasileira: evidências de um inquérito de base populacional. Revista Brasileira de Epidemiologia, 20(1), 16-29. https://dx.doi.org/10.1590/1980-5497201700010002

Gonçalves, J. G. A. et al. (2020) Terapia a laser aplicada ao tratamento da mucosite oral em pacientes com câncer. Uma análise bibliométrica. Pesquisa, Sociedade e Desenvolvimento, 9(7), 1-16. https://rsdjournal.org/index.php/rsd/article/view/4938. Acesso em: 11 mar. 2021.

Kallis, P., \& Tosti, A. (2016). Onicomicose e Onicomatricoma. Distúrbios do apêndice cutâneo, 1 (4), 209-212. https://doi.org/10.1159/000445908

Kautzky-Willer, A., Harreiter, J., \& Pacini, G. (2016). Sex and Gender Differences in Risk, Pathophysiology and Complications of Type 2 Diabetes Mellitus. Endocrine reviews, 37(3), 278-316. https://doi.org/10.1210/er.2015-1137

Liang, Y. I., Lu, L. M., Chen, Y., \& Lin, Y. K. (2016). Photodynamic therapy as an antifungal treatment. Experimental and therapeutic medicine, 12(1), 2327. https://doi.org/10.3892/etm.2016.3336

Ma, W., Si, C., Kasyanju Carrero, L. M., Liu, H. F., Yin, X. F., Liu, J., Xu, Y., \& Zhou, B. (2019). Laser treatment for onychomycosis: A systematic review and meta-analysis. Medicine, 98(48), e17948. https://doi.org/10.1097/MD.0000000000017948

Macedo, S. P. R., Mota, M. S. A., Fagundes, C. F., Souza, M. R., \& Navarro, R. S. (2021). Efeitos da fotobiomodulação no tratamento das úlceras por pressão: revisão integrativa. Research, Society and Development, 10 (2), e32810212597. https://doi.org/10.33448/rsd-v10i2.12597

Meirelles, R. M. R. (2015). Menopausa e síndrome metabólica. Arq Bras Endocrinologia Metabolismo, 2 (58), 91-96. http://dx.doi.org/10.1590/00042730000002909.

Nunes J. L. B,Filho J. S. A, Neto A. M. S,Andrade C. S,Duque B. C, Costa F. P. M et al.(2002) Doença arterial oclusiva periférica de membros inferiores em hospitais públicos de Salvador - perfil dos pacientes e do atendimento. J Vasc Br. 1(3), 201-206. https://www.jvascbras.org/article/5e2219730e8825401f6d0101/pdf/jvb-1-3-201.pdf. 
Research, Society and Development, v. 10, n. 4, e36610414099, 2021

(CC BY 4.0) | ISSN 2525-3409 | DOI: http://dx.doi.org/10.33448/rsd-v10i4.14099

Palmeira, S. C \& Pinto R. S.(2015). Perfil epidemiológico de pacientes com diabetes mellitus em Salvador, Bahia, Brasil. 2002-2012. Revista Baiana de Enfermagem, 3 (29), 240-249. http://dx.doi.org/10.18471/rbe.v29i3.13158.

Paula, L., Silva, J. L. M., \& Faria, D. P. (2012). Tratamentos Alternativos para Onicomicose: Ondas de Alta Frequência e Laser. Revista Brasileira Multidisciplinar, 15(2), 68-80. https://doi.org/10.25061/2527-2675/ReBraM/2012.v15i2.90

Picon, R. V., Dias-da-Costa, J. S., Fuchs, F. D., Olinto, M., Choudhry, N. K., \& Fuchs, S. C. (2017). Hypertension Management in Brazil: Usual Practice in Primary Care-A Meta-Analysis. International journal of hypertension, 2017, 1274168. https://doi.org/10.1155/2017/1274168

Pimentel, T. S., \& Marques, D. R. S. (2019). Atuação do enfermeiro no controle da neuropatia periférica em pacientes portadores de diabetes mellitus tipo 2. Caderno De Graduação - Ciências Biológicas e da Saúde - Unit - Sergipe, 5(2), 213. https://periodicos.set.edu.br/cadernobiologicas/article/view/6626

Portal do Enfermeiro Podiatra Brasileiro. (2020). A história da podiatria. www.podiatria.com.br.

Santos, J. B \& Duarte, F. C (2019). Onicomicoses: Identificação dos principais patógenos e manejo clínico. Rev.Iberoam.Pod, 2(1). file:///C:/Users/eupro/Downloads/9-Texto\%20do\%20artigo-52-1-10-20191115.pdf

Sarno, F., Bittencourt C. A. G., \& Oliveira, S. A. (2020). Perfil de pacientes com hipertensão arterial e/ou diabetes mellitus de unidades de Atenção Primária à Saúde. Einstein, 18, 1-6. https://doi.org/10.31744/einstein_journal/2020ao4483

Schmidt M. H, \& Pereira A. D. (2017). Laserterapia: a utilização da tecnologia na intervenção em enfermagem. Disciplinarum Scientia, 17 (3), $499-506$. https://periodicos.ufn.edu.br/index.php/disciplinarumS/article/view/2149/0

Senteio, J., Teston, E., Costa, M., Soares, V., \& Spigolon, D. (2018). Prevalence of risk factors for diabetic foot development / Prevalência de fatores de risco para o desenvolvimento de pé diabético. Revista de Pesquisa: Cuidado é Fundamental Online, 10(4), 919-925. http://dx.doi.org/10.9789/21755361.2018.v10i4.919-925

Silva J. M. T. S, Haddad M. C. F. L, Rossanus M.A, Vannuchi M. T. O., \& Marcon S. S. (2017). Fatores associados a ulcerações nos pés de pessoas com diabetes mellitus residentes em área rural. Revista Gaúcha de Enfermagem, 38(3), e68767. https://doi.org/10.1590/1983-1447.2017.03.68767

Sociedade Brasileira de Diabetes. (2020). Epidemiologia e impacto global do diabetes mellitus - Diretrizes Sociedade Brasileira de Diabetes 2019-2020. www.diabetes.gov.br. 